\title{
Synthesis of 8-aryl-substituted 4-(5-chloropyrido[4,3-d]pyrimidine- 2-yl)morpholines as intermediates of potential PI3K inhibitors via selective Suzuki-Miyaura cross-coupling reaction
}

\author{
Yanhong Chen, Chunhao Yang,* and Yuyuan Xie \\ State Key Laboratory of Drug Research, Shanghai Institute of Materia Medica, Chinese \\ Academy of Sciences, 555 Zhuchongzhi Road, Shanghai 201203, China \\ E-mail: chyang@mail.shcnc.ac.cn
}

\begin{abstract}
The selective Suzuki cross-coupling reaction of 4-(5-chloro-8-iodopyrido[4,3-d]pyrimidin-2yl)morpholine and 4-(8-bromo-5-chloropyrido[4,3-d]pyrimidin-2-yl)morpholine catalyzed by palladium has been studied during the preparation of 8-aryl-substituted 4-(5-chloropyrido[4,3d]pydimidin-2-yl)morpholines as key intermediates of potential PI3K inhibitors. This is the first report of the selective Suzuki cross-coupling reaction of fused $\pi$-deficient heterocycles containing a 2-chloro-5-halopyridine. The selectivity of the reaction is highly dependent on the nature of the palladium catalyst, solvent, base and temperature. Under optimized conditions, a variety of aryl boronic acids and even heteroaryl boronic acids were coupled in high yield and selectivity.
\end{abstract}

Keywords: Suzuki cross-coupling, regioselectivity, heterocycles, palladium catalysis, pyridopyrimidine

\section{Introduction}

Pyridopyrimidines have been extensively applied in medicinal chemistry because of their potential biological activities. ${ }^{1}$ In all possible pyridopyrimidine systems, the pyrido[2,3$d$ pyrimidine skeleton has been studied diffusely. ${ }^{2}$ Several derivatives of pyrido[4,3$d$ pyrimidine also possess a wide spectrum of biological activities, including PI3K inhibition, ${ }^{3}$ tyrosine kinase inhibition, ${ }^{4}$ and PDE-4 inhibition. ${ }^{5}$

During the course of the search for novel PI3K inhibitors, compounds possessing simple alkylamino, alkylthio or alkoxy groups at the C-5 position and aryl groups at the C-8 position of the 4-(pyrido[4,3- $d$ ]pyrimidin-2-yl)morpholine scaffold, shown in Figure 1, were designed based on the lead compound LY294002. The Suzuki-Miyaura cross-coupling reaction would be preferred to introduce an aryl group into the key intermediate I 8-bromo-2morpholinopyrido[4,3-d]pyrimidin-5(6H)-one or 8-iodo-2-morpholinopyrido[4,3-d]pyrimidin- 
5(6H)-one due to its mild reaction conditions, environmentally benign approaches and commercially available boronic acids and so on. ${ }^{6}$<smiles>O=c1cc(N2CCOCC2)oc2c(-c3ccccc3)cccc12</smiles>

LY294002<smiles>[R][X]c1ncc([Al])c2nc(N3CCOCC3)ncc12</smiles>

$\mathrm{A}=\mathrm{N}, \mathrm{S}, \mathrm{O}$

Figure 1. The lead compound LY294002 and the designed compounds.

However, it was observed that both of the above intermediates I only afforded the dehalogenated products and the conversions were very low under common Suzuki reaction conditions when reacted with phenylboronic acid. These unsatisfactory results prompted us to try to synthesize the key intermediate II 8-aryl-substituted 4-(5-chloropyrido[4,3-d]pyrimidin-2yl)morpholines via a selective Suzuki-Miyaura cross-coupling reaction.<smiles>[R][Y]c1ncc(Br)c2nc(N3CCOCC3)ncc12</smiles>

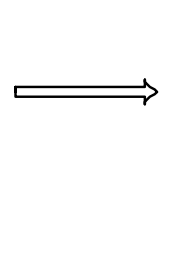<smiles>C=C</smiles><smiles>O=c1[nH]cc(Br)c2nc(N3CCOCC3)ncc12</smiles>

II<smiles>C=C</smiles><smiles>[X]c1cnc(Cl)c2cnc(N3CCOCC3)nc12</smiles>

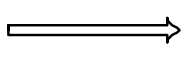<smiles>C=C</smiles><smiles>[X]c1c[nH]c(=O)c2cnc(N3CCOCC3)nc12</smiles>

I X=I, Br

Scheme 1. The key intermediates of the desired products.

To our knowledge, only a few methods have been reported to yield 5-aryl-substituted 2chloropyridine selectively from 5-bromo-2-chloropyridine ${ }^{7 \mathrm{a}-7 \mathrm{e}}$ or 2 -chloro-5-iodopyridine. ${ }^{7 \mathrm{f}, 7 \mathrm{~g}}$ However, the application of these methods is limited due to toxic or expensive organometallic 
agents such as organostannanes ${ }^{7 \mathrm{a}}$ or organosilanes. ${ }^{7 \mathrm{~b}}$ Others achieved high selectivity and yield by Suzuki cross-coupling using complex and unusual catalysts, ${ }^{7 c, 7 d}$ ligands $^{7 \mathrm{e}}$ or with the assistance of microwave heating. ${ }^{7 \mathrm{c}}$ So far, the selective Suzuki-Miyaura cross-coupling reaction of fused $\pi$-deficient heterocycles containing 2-chloro-5-iodopyridine has not been reported. Herein, we describe the selective Suzuki cross-coupling reaction of 4-(5-chloro-8iodopyrido[4,3-d]pyrimidin-2-yl)morpholine (1a) using a common palladium catalyst.

\section{Results and Discussion}

The coupling reaction of 4-(5-chloro-8-iodopyrido[4,3-d]pyrimidin-2-yl)morpholine (1a) with phenylboronic acid (2a) was examined as a model reaction to optimize the reaction conditions (Equation 1, Table 1). Initially, the effect of base was examined. We found that $\mathrm{K}_{2} \mathrm{CO}_{3}$ and $\mathrm{Cs}_{2} \mathrm{CO}_{3}$ gave moderate yields but poor selectivities. The use of $\mathrm{K}_{3} \mathrm{PO}_{4}$<smiles>[X]c1cnc(Cl)c2cnc(N3CCOCC3)nc12</smiles>

1
$\operatorname{ArB}(\mathrm{OH})_{2}$

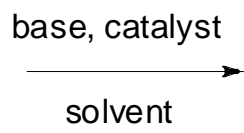

2<smiles>Clc1ncc(Br)c2nc(N3CCOCC3)ncc12</smiles>

3<smiles>[Al]c1cnc(Br)c2nc(N3CCOCC3)ncc12</smiles>

4

a: $\mathrm{X}=\mathrm{I} ; \mathrm{b}: \mathrm{X}=\mathrm{Br}$

a: $\mathrm{Ar}=\mathrm{Ph} ; \mathrm{b}: \mathrm{Ar}=p-\mathrm{MeC}_{6} \mathrm{H}_{4}$;

c: $\mathrm{Ar}=p-\mathrm{MeOC}_{6} \mathrm{H}_{4} ; \mathrm{d}: \mathrm{Ar}=3,4$-dimethoxyphenyl;

e: $\operatorname{Ar}=2,3,4$-trimethoxyphenyl; f: Ar=2-naphthyl;

$\mathrm{g}$ : Ar=benzo[d][1,3]dioxol-5-yl; h: Ar=thiophen-2-yl;

i: Ar=benzo[b]thiophen-2-yl.

\section{Equation 1}


Table 1. Palladium catalyzed Suzuki cross-coupling reaction of 4-(5-chloro-8-iodopyrido[4,3d]pyrimidin-2-yl)morpholine 1a with phenylboronic acid $\mathbf{2} \mathbf{a}^{\mathrm{a}}$

\begin{tabular}{|c|c|c|c|c|c|c|c|c|}
\hline \multirow[t]{2}{*}{ Entry } & \multirow[t]{2}{*}{ Base } & \multirow[t]{2}{*}{ Solvent } & \multirow[t]{2}{*}{ Catalyst } & \multirow[t]{2}{*}{ Time(h) } & \multirow{2}{*}{$\begin{array}{c}\text { Conversion }^{\mathrm{e}} \\
(\%)\end{array}$} & \multirow{2}{*}{$\begin{array}{c}\text { Molar ratio } \\
\mathbf{3 a} / \mathbf{4 a} \\
\end{array}$} & \multicolumn{2}{|c|}{ yield $^{\mathrm{f}}(\%)$} \\
\hline & & & & & & & $\mathbf{3 a}$ & $4 a$ \\
\hline 1 & $\mathrm{~K}_{2} \mathrm{CO}_{3}$ & dioxane & $\mathrm{Pd}\left(\mathrm{PPh}_{3}\right)_{4}$ & 8 & 100 & 65:35 & 39 & 21 \\
\hline 2 & $\mathrm{Cs}_{2} \mathrm{CO}_{3}$ & dioxane & $\mathrm{Pd}\left(\mathrm{PPh}_{3}\right)_{4}$ & 8 & 100 & 39:61 & 25 & 39 \\
\hline 3 & $\mathrm{~K}_{3} \mathrm{PO}_{4}$ & dioxane & $\mathrm{Pd}\left(\mathrm{PPh}_{3}\right)_{4}$ & 8 & 100 & $79: 21$ & 68 & 18 \\
\hline 4 & $\mathrm{NaOH}$ & dioxane & $\mathrm{Pd}\left(\mathrm{PPh}_{3}\right)_{4}$ & 8 & 100 & 95:5 & 79 & 4 \\
\hline 5 & KF & dioxane & $\mathrm{Pd}\left(\mathrm{PPh}_{3}\right)_{4}$ & 10 & 98 & $96: 4$ & 75 & 3 \\
\hline $6^{\mathrm{b}}$ & $\mathrm{NaOH}$ & dioxane & $\mathrm{Pd}\left(\mathrm{PPh}_{3}\right)_{4}$ & 8 & 100 & $91: 9$ & 70 & 7 \\
\hline 7 & $\mathrm{NaOH}$ & toluene & $\mathrm{Pd}\left(\mathrm{PPh}_{3}\right)_{4}$ & 8 & 100 & $95: 5$ & 63 & 3 \\
\hline 8 & $\mathrm{NaOH}$ & DMF & $\mathrm{Pd}\left(\mathrm{PPh}_{3}\right)_{4}$ & 8 & 100 & $51: 49$ & 15 & 14 \\
\hline 9 & $\mathrm{NaOH}$ & dioxane & $\mathrm{PdCl}_{2}\left(\mathrm{PPh}_{3}\right)_{2}$ & 8 & 100 & 68:32 & 46 & 22 \\
\hline 10 & $\mathrm{NaOH}$ & dioxane & $\mathrm{PdCl}_{2}(\mathrm{dppf})$ & 8 & 100 & 63:37 & 40 & 23 \\
\hline 11 & $\mathrm{NaOH}$ & dioxane & $\operatorname{Pd}(\mathrm{OAc})_{2}{ }^{\mathrm{c}}$ & 8 & 100 & 90:10 & 51 & 6 \\
\hline $12^{\mathrm{d}}$ & $\mathrm{NaOH}$ & dioxane & $\mathrm{Pd}\left(\mathrm{PPh}_{3}\right)_{4}$ & 48 & 84 & 99:1 & 61 & 1 \\
\hline
\end{tabular}

${ }^{a}$ Reaction conditions: 1a (0.133 mmol), 2a (1.5 equiv.), [Pd] (0.05 equiv.), base (3 equiv.), and solvent $(5 \mathrm{~mL}) .{ }^{\mathrm{b}} 2 \mathrm{a}$ (1 equiv.). ${ }^{\mathrm{c}} 0.1$ equiv. of $\mathrm{PPh}_{3}$ was used as ligand. ${ }^{\mathrm{d}}$ The temperature was 80 ${ }^{\circ} \mathrm{C}$. ${ }^{\mathrm{e}}$ Average conversion of two runs based on HPLC results. ${ }^{\mathrm{f}}$ Average yield of two runs based on HPLC results.

delivered a significant increase in the yield of the mono substituted product (3a). $\mathrm{NaOH}$ and $\mathrm{KF}$ gave good yields and selectivities. Considering that KF extended the reaction time and reduced the conversion and yield, $\mathrm{NaOH}$ was employed for the next set of experiments, despite that $\mathrm{KF}$ slightly increased the selectivity compared to $\mathrm{NaOH}$. Reducing the amount of phenylboronic acid to 1 equivalent did not improve the selectivity.

The solvent was also found to have a profound impact on the reaction. When the reaction was carried out in toluene instead of dioxane, the selectivity did not change but the yield of compound (3a) decreased from $79 \%$ to $63 \%$. It was noted that the polar solvent DMF remarkably reduced the yield and selectivity.

Optimization of the palladium catalyst was then carried out. The examined palladium catalysts included $\mathrm{PdCl}_{2}\left(\mathrm{PPh}_{3}\right)_{2}, \mathrm{PdCl}_{2}(\mathrm{dppf})$, and $\mathrm{Pd}(\mathrm{OAc})_{2}$ in the presence of $\mathrm{PPh}_{3} . \mathrm{PdCl}_{2}\left(\mathrm{PPh}_{3}\right)_{2}$ or $\mathrm{PdCl}_{2}$ (dppf) gave a moderate yield of $\mathbf{3 a} / \mathbf{4 a}$. Obviously, all of these catalysts did not produce any advantages over the $\mathrm{Pd}\left(\mathrm{PPh}_{3}\right)_{4}$ originally used.

Finally, we investigated the possibility of improving the selectivity by lowering the reaction temperature. Although lowering the temperature to $80^{\circ} \mathrm{C}$ led to a significant improvement in selectivity, the starting material could not be converted completely even by prolonging the reaction time to $48 \mathrm{~h}$. 
Under the optimized reaction conditions $\left(\mathrm{NaOH}, \mathrm{Pd}\left(\mathrm{PPh}_{3}\right)_{4}\right.$, dioxane, $\left.110^{\circ} \mathrm{C}\right)$, we investigated the scope and the limitations of the reaction employing a variety of aryl boronic acids with 4-(5chloro-8-iodopyrido[4,3- $d$ ]pyrimidin-2-yl)morpholine (1a) or 4-(8-bromo-5-chloropyrido[4,3d]pyrimidin-2-yl)morpholine (1b) as coupling partners. The results are summarized in Table 2.

In general, the more electron-deficient site on the haloheterocycles is the preferred position for coupling. ${ }^{8}$ Accordingly, related templates bearing identical halogens at both the C-5 and C-8 positions will react at the more activated C-5 position first. However, in our study, selective coupling at the C-8 position of compound (1a) was observed, except for 2,3,4trimethoxyphenylboronic acid (entry 4), demonstrating that the reactivity of the iodine dominated over the electronic effect. Substitution with electron-donating groups at the paraposition on phenyl boronic acid gave very good yield and selectivity, such as methyl, methoxy and even 3,4-dimethoxy (entries 1-3). The bulky 2-naphthyl (entry 5) and benzo[d][1,3]dioxol5-ylboronic acid (entry 6) reacted smoothly and gave very excellent selectivity. To our delight, heterocycles such as thiophen-2-ylboronic acid (entry 7) and benzo[b]thiophen-2-ylboronic acid (entry 8) gave only monosubstituted products (3h) and (3i) respectively. As expected, the selectivity of compound (1b) was poor (entry 9 and 10). When (1b) was reacted with phenyl boronic acid, the chlorine was substituted to afford 4-(8-bromo-5-phenylpyrido[4,3- $d$ ]pyrimidin2-yl)morpholine (3a', entry 9). However, when it reacted with $p$-methoxyphenyl boronic acid, the bromine atom was replaced to give 4-(5-chloro-8-(4-methoxyphenyl)pyrido[4,3- $d$ ]pyrimidin2-yl)morpholine (3c, entry 10). The difference of reactivity between chlorine and bromine was offset by the electronic effects. The selectivity also depended on the substrate.

Table 2. Palladium catalyzed Suzuki cross-coupling reaction of 4-(5-chloro-8-iodopyrido [4,3-d]pyrimidin-2-yl)morpholine or 4-(8-bromo-5-chloropyrido[4,3-d]pyrimidin-2yl)morpholine with arylboronic acid ${ }^{\mathrm{a}}$

\begin{tabular}{|c|c|c|c|c|c|c|c|}
\hline \multirow[t]{2}{*}{ Entry } & \multirow[t]{2}{*}{1} & \multirow[t]{2}{*}{$\operatorname{ArB}(\mathrm{OH})_{2}$} & \multirow{2}{*}{$\begin{array}{l}\text { Time } \\
\text { (h) }\end{array}$} & \multirow{2}{*}{$\begin{array}{c}\text { Conversion } \\
\text { (\%) }\end{array}$} & \multirow{2}{*}{$\begin{array}{c}\text { Molar } \\
\text { ratio } \\
\mathbf{3} / \mathbf{4} \\
\end{array}$} & \multicolumn{2}{|c|}{ Yield $(\%)^{b}$} \\
\hline & & & & & & 3 & 4 \\
\hline 1 & (1a) & $(2 b)$ & 6 & 100 & $94: 6$ & $\begin{array}{c}94 \\
(3 \mathbf{b})\end{array}$ & $\begin{array}{c}6 \\
(4 b)\end{array}$ \\
\hline 2 & (1a) & (2c) & 6 & 100 & $97: 3$ & $\begin{array}{c}96 \\
(\mathbf{3 c})\end{array}$ & $\begin{array}{c}3 \\
(4 c)\end{array}$ \\
\hline 3 & (1a) & (2d) & 6 & 100 & $94: 6$ & $\begin{array}{c}75 \\
(\mathbf{3 d})\end{array}$ & $\begin{array}{c}5 \\
(4 d)\end{array}$ \\
\hline 4 & (1a) & $(2 e)$ & 6 & 100 & $42: 58$ & $\begin{array}{c}29 \\
(\mathbf{3 e})\end{array}$ & $\begin{array}{c}29 \\
(4 e)\end{array}$ \\
\hline 5 & (1a) & (2f) & 6 & 100 & $98: 2$ & $\begin{array}{c}97 \\
(\mathbf{3 f})\end{array}$ & $\begin{array}{c}2 \\
(4 f)\end{array}$ \\
\hline
\end{tabular}


Table 2. Continued

\begin{tabular}{|c|c|c|c|c|c|c|c|}
\hline 6 & (1a) & $(2 \mathrm{~g})$ & 6 & 100 & $98: 2$ & $\begin{array}{l}79 \\
(\mathbf{3 g})\end{array}$ & $\begin{array}{l}2 \\
(4 g)\end{array}$ \\
\hline 7 & (1a) & (2h) & 24 & 91 & 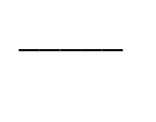 & $\begin{array}{l}47 \\
(\mathbf{3 h})\end{array}$ & 0 \\
\hline 8 & (1a) & $(2 \mathbf{i})$ & 24 & 56 & 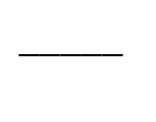 & $\begin{array}{l}35 \\
(\mathbf{3 i})\end{array}$ & 0 \\
\hline 9 & (1b) & (2a) & 6 & 100 & $44: 56$ & $\begin{array}{l}29 \\
\left(\mathbf{3} \mathbf{a}^{\prime}\right)^{\mathrm{c}}\end{array}$ & $\begin{array}{l}37 \\
\mathbf{( 4 a )}\end{array}$ \\
\hline 10 & (1b) & $(2 \mathrm{c})$ & 24 & 90 & $67: 33$ & $\begin{array}{l}30 \\
(\mathbf{3 c})\end{array}$ & $\begin{array}{l}15 \\
(4 c)\end{array}$ \\
\hline
\end{tabular}

${ }^{a}$ Reaction conditions: 1 (1.6 mmol), 2 (2.4 mmol), $\mathrm{NaOH}(4.8 \mathrm{mmol}), \mathrm{Pd}\left(\mathrm{PPh}_{3}\right)_{4}(0.08 \mathrm{mmol})$, and dioxane $(60 \mathrm{~mL}), \quad 110{ }^{\circ} \mathrm{C}$. ' Isolated yield. ' $3 \mathrm{a}$ ' is 4-(8-bromo-5-phenylpyrido[4,3d]pyrimidin-2-yl)morpholine.

\section{Conclusions}

In summary, we have developed an efficient and selective protocol for the coupling reaction of 4-(5-chloro-8-iodopyrido[4,3-d]pyrimidin-2-yl)morpholine with various aryl boronic acids to construct pyrido[4,3-d]pyrimidines. Under the optimized conditions $\left(\mathrm{Pd}\left(\mathrm{PPh}_{3}\right)_{4}, \mathrm{NaOH}\right.$, dioxane, $110{ }^{\circ} \mathrm{C}$ ), most of the aryl boronic acids underwent the coupling reaction smoothly in good yield and high selectivity. However, the optimized conditions did not fit for 4-(8-bromo-5chloropyrido[4,3- $d$ ]pyrimidin-2-yl)morpholine. This would be a useful probe to synthesize pyrido[4,3-d]pyrimidines or related fused $\pi$-deficient pyridines.

\section{Experimental Section}

General Procedures. All experiments were carried out under a nitrogen atmosphere. Palladium complexes, aryl boronic acids were obtained from commercial sources and used without further purification. 4-(5-chloro-8-iodopyrido[4,3-d]pyrimidin-2-yl)morpholine (1a) and 4-(8-bromo-5chloropyrido[4,3- $d$ ]pyrimidin-2-yl)morpholine (1b) were synthesized in our laboratory. HPLC analyses were performed on an Agilent 1100 apparatus equipped with a DAD detector and an Agilent ZORBAX Eclipse XDB C-18 $(4.6 \times 150 \mathrm{~mm}, 5 \mu \mathrm{m})$ column. The condition of HPLC was as follows: flow rate: $0.8 \mathrm{~mL} / \mathrm{min}$; solvent gradient: 0-10 min, $\mathrm{H}_{2} \mathrm{O}: \mathrm{CH}_{3} \mathrm{CN}=50: 50$; $12 \mathrm{~min}-30 \mathrm{~min}, \mathrm{H}_{2} \mathrm{O}: \mathrm{CH}_{3} \mathrm{CN}=40: 60$; column temperature: $25^{\circ} \mathrm{C}$; wavelength: $275 \mathrm{~nm} .{ }^{1} \mathrm{H}$ and ${ }^{13} \mathrm{C}$ NMR spectra were recorded on Varian Mercury-300 and Varian Mercury-400 spectrometers. IR spectra were recorded on a Perkin-Elmer 983G infrared spectrometer using $\mathrm{KBr}$ pellets. 
LRMS and HRMS spectra were measured on Finnigan MAT 95 spectrometer using electron impact (EI) and an electron beam of $70 \mathrm{eV}$. Melting points were measured by Büchi 510 melting point apparatus and were uncorrected.

\section{General procedure for the Suzuki cross-coupling reaction}

A flask charged with 4-(5-chloro-8-iodopyrido[4,3-d]pyrimidin-2-yl)morpholine (1.6 mmol), aryl boronic acid (2.4 mmol), $2 \mathrm{M} \mathrm{NaOH}(2.4 \mathrm{~mL}, 4.8 \mathrm{mmol}), \mathrm{Pd}\left(\mathrm{PPh}_{3}\right)_{4}(0.08 \mathrm{mmol})$ in dioxane $(60 \mathrm{~mL})$ was evacuated and backfilled with nitrogen. And the reaction mixture was refluxed for 6-24 h under a nitrogen atmosphere. After cooling to room temperature, the mixture was poured into water, filtered and dried to afford yellow solid. The crude solid was purified by column chromatography on silica gel (petroleum ether/ethyl acetate or petroleum ether/chloroform) to afford the products 3 and 4 .

4-(5-Chloro-8-phenylpyrido[4,3-d]pyrimidin-2-yl)morpholine (3a). Yellow solid, mp 186$187^{\circ} \mathrm{C}$. IR (KBr): 2918, 1599, 1579, 1514, 1124, 860, $692 \mathrm{~cm}^{-1} .{ }^{1} \mathrm{H}$ NMR (300 MHz, $\left.\mathrm{CDCl}_{3}\right): \delta$ = 9.40 (s, $1 \mathrm{H}), 8.39$ (s, $1 \mathrm{H}), 7.69$ (d, $J=6.9 \mathrm{~Hz}, 2 \mathrm{H})$, 7.50-7.39 (m, $3 \mathrm{H}), 3.95$ (br s, $4 \mathrm{H}), 3.76$ (br s, $4 \mathrm{H}$ ). ${ }^{13} \mathrm{C}$ NMR $\left(100 \mathrm{MHz}, \mathrm{CDCl}_{3}\right): \delta=161.2,160.2,155.2,150.5,148.6,134.5,130.8$, 130.0, 128.1, 128.0, 113.7, 66.8, 44.4. LRMS: $\mathrm{m} / \mathrm{z}(\%)=328(33)[\mathrm{M}+2]^{+}, 326(100)[\mathrm{M}]^{+}$. HRMS calcd for $\mathrm{C}_{17} \mathrm{H}_{15} \mathrm{ClN}_{4} \mathrm{O}$ : 326.0934; found: 326.0930 .

4-(8-Bromo-5-phenylpyrido[4,3-d]pyrimidin-2-yl)morpholine (3a'). Yellow solid, mp 196$200{ }^{\circ} \mathrm{C}$. IR (KBr): 2968, 1597, 1558, 1510, 1113, $704 \mathrm{~cm}^{-1} .{ }^{1} \mathrm{H}$ NMR $\left(300 \mathrm{MHz}, \mathrm{CDCl}_{3}\right): \delta=$ 9.11 (s, 1 H), 8.85 (s, 1 H), 7.67-7.64 (m, 2 H), 7.55-7.52 (m, 3 H), 4.07 (br s, 4 H), 3.82 (br s, 4 H). ${ }^{13} \mathrm{C}$ NMR $\left(100 \mathrm{MHz}, \mathrm{CDCl}_{3}\right): \delta=162.7,160.5,160.0,153.8,151.4,136.8,129.9,129.6$, 128.7, 116.4, 114.7, 66.8, 44.4. LRMS: $\mathrm{m} / \mathrm{z}(\%)=372(100)[\mathrm{M}+2]^{+}, 370(100)[\mathrm{M}]^{+}$. HRMS calcd for $\mathrm{C}_{17} \mathrm{H}_{15} \mathrm{BrN}_{4} \mathrm{O}$ : 370.0429 ; found: 370.0442 .

4-(5,8-Diphenylpyrido[4,3-d]pyrimidin-2-yl)morpholine (4a). Yellow solid, mp 204-206 ${ }^{\circ} \mathrm{C}$. IR (KBr): 2958, 1595, 1578, 1514, 1115, $700 \mathrm{~cm}^{-1} .{ }^{1} \mathrm{H}$ NMR (300 MHz, $\left.\mathrm{CDCl}_{3}\right): \delta=9.24$ (s, 1 H), 8.75 (s, $1 \mathrm{H})$, 7.83-7.80 (m, 2 H), 7.74-7.71 (m, $2 \mathrm{H})$, 7.59-7.40 (m, $6 \mathrm{H}), 3.84$ (br s, $4 \mathrm{H}$ ), $3.76(\mathrm{t}, J=4.5 \mathrm{~Hz}, 4 \mathrm{H}) .{ }^{13} \mathrm{C}$ NMR $\left(100 \mathrm{MHz}, \mathrm{CDCl}_{3}\right): \delta=162.6,160.6,159.6,153.9,149.5$, 137.5, 135.5, 130.2, 130.0, 129.4, 129.3, 128.6, 128.1, 127.7, 113.6, 66.8, 44.3. LRMS: m/z (\%) $=368(100)[\mathrm{M}]^{+}$. HRMS calcd for $\mathrm{C}_{23} \mathrm{H}_{20} \mathrm{~N}_{4} \mathrm{O}$ : 368.1637, found: 368.1642 .

4-(5-Chloro-8-p-tolylpyrido[4,3-d]pyrimidin-2-yl)morpholine (3b). Yellow solid, mp 231-233 ${ }^{\circ} \mathrm{C}$. IR (KBr): 2928, 1600, 1577, 1506, 1122, $820 \mathrm{~cm}^{-1} .{ }^{1} \mathrm{H}$ NMR (300 MHz, $\mathrm{CDCl}_{3}$ ): $\delta=9.40$ (s, $1 \mathrm{H}$ ), 8.38 (s, $1 \mathrm{H}$ ), 7.60 (d, $J=8.0 \mathrm{~Hz}, 2 \mathrm{H}$ ), 7.29 (d, $J=8.0 \mathrm{~Hz}, 2 \mathrm{H}$ ), 3.95 (br s, $4 \mathrm{H}$ ), 3.76 (t, $J$ $=4.8 \mathrm{~Hz}, 4 \mathrm{H}), 2.43(\mathrm{~s}, 3 \mathrm{H}) .{ }^{13} \mathrm{C}$ NMR $\left(100 \mathrm{MHz}, \mathrm{CDCl}_{3}\right): \delta=161.2,160.2,155.2,150.1,148.4$, 137.9, 131.5, 130.7, 129.8, 128.9, 113.7, 66.8, 44.4, 21.3. LRMS: $\mathrm{m} / \mathrm{z}(\%)=342(33)[\mathrm{M}+2]^{+}$, 340 (100) [M] ${ }^{+}$. HRMS calcd for $\mathrm{C}_{18} \mathrm{H}_{17} \mathrm{ClN}_{4} \mathrm{O}$ : 340.1091; found: 340.1077 .

4-(5,8-Di-p-tolylpyrido[4,3-d]pyrimidin-2-yl)morpholine (4b). Yellow solid, mp 200-201 ${ }^{\circ} \mathrm{C}$. IR (KBr): 2960, 1600, 1556, 1506, 1109, 812 $\mathrm{cm}^{-1} .{ }^{1} \mathrm{H}$ NMR (300 MHz, $\left.\mathrm{CDCl}_{3}\right): \delta=9.25$ (s, 1 H), 8.72 (s, $1 \mathrm{H}$ ), 7.71 (d, $J=8.0 \mathrm{~Hz}, 2 \mathrm{H}), 7.62$ (d, $J=8.0 \mathrm{~Hz}, 2 \mathrm{H}), 7.35$ (d, $J=8.0 \mathrm{~Hz}, 2 \mathrm{H}$ ), 7.31 (d, $J=8.0$ Hz, 2 H), 3.95 (br s, 4 H), 3.77 (t, $J=4.2$ Hz, 4 H), 2.47 (s, 3 H), 2.44 (s, 3 H). 
${ }^{13} \mathrm{C}$ NMR (100 MHz, $\left.\mathrm{CDCl}_{3}\right): \delta=162.6,160.4,159.5,154.0,149.4,139.4,137.4,134.7,132.6$, 130.0, 129.3, 128.9, 128.8, 113.6, 66.8, 44.3, 21.4, 21.3. LRMS: m/z (\%) = $396(100)[\mathrm{M}]^{+}$. HRMS calcd for $\mathrm{C}_{25} \mathrm{H}_{24} \mathrm{~N}_{4} \mathrm{O}$ : 396.1950; found: 396.1942.

4-(5-Chloro-8-(4-methoxyphenyl)pyrido[4,3-d]pyrimidin-2-yl)morpholine (3c). Yellow solid, mp 205-206 ${ }^{\circ} \mathrm{C}$. IR (KBr): 2943, 1599, 1579, 1504, 1120, 827 $\mathrm{cm}^{-1} .{ }^{1} \mathrm{H}$ NMR (300 MHz, $\left.\mathrm{CDCl}_{3}\right)$ : $\delta=9.40$ (s, 1 H), 9.36 (s, 1 H), 7.65 (d, $J=8.5$ Hz, 2 H), 7.00 (d, $J=8.5$ Hz, 2 H), 3.96 (br s, 4 $\mathrm{H}), 3.88$ (s, $3 \mathrm{H}), 3.76$ (t, $J=4.5 \mathrm{~Hz}, 4 \mathrm{H}) .{ }^{13} \mathrm{C} \mathrm{NMR}\left(100 \mathrm{MHz}, \mathrm{CDCl}_{3}\right): \delta=161.2,160.2,159.5$, 155.1, 149.8, 148.1, 131.1, 130.4, 126.7, 113.6, 66.7, 55.3, 44.4. LRMS: m/z (\%) = 358 (33) $[\mathrm{M}+2]^{+}, 356$ (100) $[\mathrm{M}]^{+}$. HRMS calcd for $\mathrm{C}_{18} \mathrm{H}_{17} \mathrm{ClN}_{4} \mathrm{O}_{2}$ : 356.1040; found: 356.1033.

4-(5,8-Bis(4-methoxyphenyl)pyrido[4,3-d]pyrimidin-2-yl)morpholine (4c). Yellow solid, mp 234-236 ${ }^{\circ} \mathrm{C}$. IR (KBr): 2960, 1595, 1574, 1508, 1180, $847 \mathrm{~cm}^{-1} .{ }^{1} \mathrm{H}$ NMR $\left(300 \mathrm{MHz}, \mathrm{CDCl}_{3}\right): \delta=$ 9.25 (s, $1 \mathrm{H}), 8.69$ (s, $1 \mathrm{H}), 7.76$ (d, $J=8.4 \mathrm{~Hz}, 2 \mathrm{H}), 7.68$ (d, $J=8.4 \mathrm{~Hz}, 2 \mathrm{H}), 7.06$ (t, $J=9.5 \mathrm{~Hz}$, $4 \mathrm{H}), 3.95$ (br s, $4 \mathrm{H}), 3.90$ (s, $3 \mathrm{H}), 3.89$ (s, $3 \mathrm{H}), 3.77$ (t, $J=4.5 \mathrm{~Hz}, 4 \mathrm{H}) .{ }^{13} \mathrm{C} \mathrm{NMR}(100 \mathrm{MHz}$, $\left.\mathrm{CDCl}_{3}\right): \delta=162.6,160.6,159.7,159.5,159.2,153.9,149.1,131.4,131.2,130.1,128.4,127.9$, 114.1, 113.5, 66.8, 55.4, 55.3, 44.3.

LRMS: $\mathrm{m} / \mathrm{z}(\%)=428(100)[\mathrm{M}]^{+}$. HRMS calcd for $\mathrm{C}_{25} \mathrm{H}_{24} \mathrm{~N}_{4} \mathrm{O}_{3}$ : 428.1848; found: 428.1854.

4-(5-Chloro-8-(3,4-dimethoxyphenyl)pyrido[4,3-d]pyrimidin-2-yl)morpholine (3d).

Yellow solid, mp 215-218 ${ }^{\circ} \mathrm{C}$. IR (KBr): 2929, 1600, 1571, 1508, 1118, $947 \mathrm{~cm}^{-1} .{ }^{1} \mathrm{H}$ NMR (300 $\mathrm{MHz}, \mathrm{CDCl}_{3}$ ): $\delta=9.39$ (s, $1 \mathrm{H}$ ), 8.37 (s, $1 \mathrm{H}$ ), 7.32 (d, $J=1.4 \mathrm{~Hz}, 1 \mathrm{H}$ ), 7.21 (dd, $J=8.3,1.4 \mathrm{~Hz}$, $1 \mathrm{H}), 6.97$ (d, $J=8.3 \mathrm{~Hz}, 1 \mathrm{H}$ ), 3.95 (br s, $4 \mathrm{H}$ ), 3.94 (s, $3 \mathrm{H}), 3.89$ (s, $3 \mathrm{H}$ ), 3.75 (t, J = $4.5 \mathrm{~Hz}, 4$ $\mathrm{H}) .{ }^{13} \mathrm{C}$ NMR (100 MHz, $\left.\mathrm{CDCl}_{3}\right): \delta=161.3,160.2,155.1,150.0,149.1,148.3,130.5,127.1$, 122.2, 113.7, 113.5, 110.9, 66.8, 55.9, 55.8, 44.4. LRMS: m/z (\%) = 388 (33) [M+2] $]^{+}, 386(100)$ $[\mathrm{M}]^{+}$. HRMS calcd for $\mathrm{C}_{19} \mathrm{H}_{19} \mathrm{ClN}_{4} \mathrm{O}_{3}$ : 386.1146; found: 386.1148.

4-(5,8-Bis (3,4-dimethoxyphenyl)pyrido[4,3-d]pyrimidin-2-yl)morpholine (4d). Yellow solid, mp 226-229 ${ }^{\circ} \mathrm{C}$. IR (KBr): 2950, 1600, 1583, 1521, 1145, $766 \mathrm{~cm}^{-1} .{ }^{1} \mathrm{H}$ NMR (300 MHz, $\mathrm{CDCl}_{3}$ ): $\delta=9.28$ (s, 1 H), 8.72 (s, 1 H), 7.45 (d, $J=2.0 \mathrm{~Hz}, 1 \mathrm{H}$ ), 7.34 (d, $J=2.0 \mathrm{~Hz}, 1 \mathrm{H}$ ), 7.31 (dd, $J=$ 8.4, $2.0 \mathrm{~Hz}, 1 \mathrm{H}$ ), 7.22 (dd, $J=8.4,2.0 \mathrm{~Hz}, 1 \mathrm{H}$ ), 7.02 (dd, $J=8.4,1.1 \mathrm{~Hz}, 2 \mathrm{H}$ ), 3.98-3.96 (br s, $4 \mathrm{H}), 3.98$ (s, $3 \mathrm{H}), 3.97$ (s, $3 \mathrm{H}), 3.96$ (s, $3 \mathrm{H}), 3.92$ (s, $3 \mathrm{H}), 3.76$ (t, $J=4.5 \mathrm{~Hz}, 4 \mathrm{H}) .{ }^{13} \mathrm{C} \mathrm{NMR}$ $\left(100 \mathrm{MHz}, \mathrm{CDCl}_{3}\right): \delta=162.7,159.9,159.5,154.0,150.2,149.3,149.1,148.7,148.2,130.2$, 128.5, 128.2, 123.3, 122.2, 113.7, 113.5, 112.7, 110.9, 110.7, 66.7, 56.0, 55.9, 55.8, 44.3. LRMS: $\mathrm{m} / \mathrm{z}(\%)=488(100)[\mathrm{M}]^{+}$. HRMS calcd for $\mathrm{C}_{27} \mathrm{H}_{28} \mathrm{~N}_{4} \mathrm{O}_{5}$ : 488.2059; found: 488.2056.

4-(5-Chloro-8-(2,3,4-trimethoxyphenyl)pyrido[4,3-d]pyrimidin-2-yl)morpholine

(3e). Yellow solid, mp $164-165{ }^{\circ} \mathrm{C}$. IR (KBr): 2933, 1599, 1570, 1521, 1099, $868 \mathrm{~cm}^{-1} .{ }^{1} \mathrm{H}$ NMR (300 $\mathrm{MHz}, \mathrm{CDCl}_{3}$ ): $\delta=9.38$ (s, $1 \mathrm{H}$ ), 8.30 (s, $\left.1 \mathrm{H}\right), 7.03$ (d, $\left.J=8.5 \mathrm{~Hz}, 1 \mathrm{H}\right), 6.74$ (d, $J=8.5 \mathrm{~Hz}, 1 \mathrm{H}$ ), 3.95 (br s, 4 H), 3.93 (s, 3 H), 3.90 (s, 3 H), 3.71 (br s, 4 H), 3.68 (s, 3 H). ${ }^{13} \mathrm{C}$ NMR (100 MHz, $\left.\mathrm{CDCl}_{3}\right): \delta=161.0,160.1,156.1,153.9,152.2,150.3,149.3,142.0,128.6,126.0,121.3,113.6$, 106.7, 66.7, 61.1, 60.8, 56.0, 44.3. LRMS: $\mathrm{m} / \mathrm{z}(\%)=418(33)[\mathrm{M}+2]^{+}, 416(100)[\mathrm{M}]^{+}$. HRMS calcd for $\mathrm{C}_{20} \mathrm{H}_{21} \mathrm{ClN}_{4} \mathrm{O}_{4}$ : 416.1251; found: 416.1252 .

4-(5,8-Bis (2,3,4-trimethoxyphenyl)pyrido[4,3-d]pyrimidin-2-yl)morpholine (4e). Yellow solid, mp 171-172 ${ }^{\circ} \mathrm{C}$. IR (KBr): 2933, 2843, 1593, 1562, 1486, 1097, $800 \mathrm{~cm}^{-1} .{ }^{1} \mathrm{H}$ NMR (300 
MHz, $\left.\mathrm{CDCl}_{3}\right): \delta=8.95$ (s, $\left.1 \mathrm{H}\right), 8.64$ (s, $\left.1 \mathrm{H}\right), 7.23$ (d, $\left.J=8.2 \mathrm{~Hz}, 1 \mathrm{H}\right), 7.15$ (d, $J=8.6 \mathrm{~Hz}, 1 \mathrm{H}$ ), 6.85 (d, $J=8.6 \mathrm{~Hz}, 1 \mathrm{H}), 6.78$ (d, $J=8.2 \mathrm{~Hz}, 1 \mathrm{H}), 3.97$ (s, $3 \mathrm{H}), 3.95$ (s, $6 \mathrm{H}), 3.93$ (s, $3 \mathrm{H}), 3.88$ (br s, $4 \mathrm{H}$ ), 3.75 (s, $3 \mathrm{H}), 3.72$ (t, $J=4.5 \mathrm{~Hz}, 4 \mathrm{H}), 3.63$ (s, $3 \mathrm{H}) \cdot{ }^{13} \mathrm{C}$ NMR $\left(100 \mathrm{MHz}, \mathrm{CDCl}_{3}\right): \delta$ = 163.1, 159.6, 157.8, 154.7, 154.1, 153.6, 152.4, 151.6, 150.2, 142.0, 127.3, 126.2, 125.9, 124.7, 122.5, 114.2, 107.8, 106.6, 66.8, 61.5, 61.2, 61.1, 60.8, 56.1, 56.0, 44.2. LRMS: $\mathrm{m} / \mathrm{z}(\%)=548$ (100) $[\mathrm{M}]^{+}$. HRMS calcd for $\mathrm{C}_{29} \mathrm{H}_{32} \mathrm{~N}_{4} \mathrm{O}_{7}$ : 548.2271; found: 548.2273.

4-(5-Chloro-8-(naphthalen-2-yl)pyrido[4,3-d]pyrimidin-2-yl)morpholine (3f). Yellow solid, mp 191-194 ${ }^{\circ} \mathrm{C}$. IR (KBr): 2929, 1595, 1575, 1513, 1117, $812 \mathrm{~cm}^{-1} .{ }^{1} \mathrm{H}$ NMR (300 MHz, $\left.\mathrm{CDCl}_{3}\right)$ : $\delta=9.43$ (s, $1 \mathrm{H}), 8.50$ (s, $1 \mathrm{H}), 8.13$ (s, $1 \mathrm{H}), 7.94-7.82$ (m, 4 H), 7.54-7.51 (m, $2 \mathrm{H}), 3.98$ (br s, 4 $\mathrm{H}), 3.75$ (br s, $4 \mathrm{H}$ ). ${ }^{13} \mathrm{C}$ NMR (100 MHz, $\mathrm{CDCl}_{3}$ ): $\delta=161.3,160.3,155.4,150.5,148.8,133.3$, 132.9, 132.2, 130.8, 129.0, 128.2, 128.0, 127.6, 127.3, 126.4, 126.3, 113.7, 66.8, 44.4. LRMS: $\mathrm{m} / \mathrm{z}(\%)=378(33)[\mathrm{M}+2]^{+}, 376(100)[\mathrm{M}]^{+}$. HRMS calcd for $\mathrm{C}_{21} \mathrm{H}_{17} \mathrm{ClN}_{4} \mathrm{O}: 376.1091$; found: 376.1092 .

4-(5,8-Di(naphthalen-2-yl)pyrido[4,3-d]pyrimidin-2-yl)morpholine (4f). Yellow solid, mp 215-217 ${ }^{\circ} \mathrm{C}$. IR (KBr): 2852, 1589, 1552, 1512, 1114, $818 \mathrm{~cm}^{-1} .{ }^{1} \mathrm{H}$ NMR (300 MHz, $\left.\mathrm{CDCl}_{3}\right): \delta$ = 9.35 (s, $1 \mathrm{H}), 8.91$ (s, $1 \mathrm{H}), 8.26(\mathrm{~s}, 1 \mathrm{H}), 8.21$ (s, $1 \mathrm{H}), 8.06-7.93(\mathrm{~m}, 8 \mathrm{H}), 7.57$ (s, $4 \mathrm{H}), 3.97$ (br s, $4 \mathrm{H}$ ), 3.77 (br s, $4 \mathrm{H}) .{ }^{13} \mathrm{C}$ NMR (100 MHz, $\left.\mathrm{CDCl}_{3}\right): \delta=162.7,160.6,159.6,154.2,149.8$, 134.8, 133.6, 133.4, 133.3, 133.0, 132.8, 130.0, 129.3, 129.0, 128.6, 128.4, 128.2, 127.8, 127.6, 127.2, 127.1, 127.0, 126.7, 126.2, 126.1, 113.8, 66.8, 44.4. LRMS: m/z (\%) = $468(100)[\mathrm{M}]^{+}$. HRMS calcd for $\mathrm{C}_{31} \mathrm{H}_{24} \mathrm{~N}_{4} \mathrm{O}: 468.1950$; found: 468.1967.

\section{4-(8-(Benzo[d][1,3]dioxol-5-yl)-5-chloropyrido[4,3-d]pyrimidin-2-yl)morpholine}

(3g). Yellow solid, mp 242-245 ${ }^{\circ} \mathrm{C}$. IR (KBr): 2904, 1599, 1571, 1514, 1117, 1041, $816 \mathrm{~cm}^{-1} .{ }^{1} \mathrm{H}$ NMR (300 MHz, $\left.\mathrm{CDCl}_{3}\right): \delta=9.38$ (s, $\left.1 \mathrm{H}\right), 8.33$ (s, $\left.1 \mathrm{H}\right), 7.24$ (d, $J=2.1 \mathrm{~Hz}, 1 \mathrm{H}$ ), 7.13 (dd, $J=$ 8.0, $2.1 \mathrm{~Hz}, 1 \mathrm{H}$ ), 6.91 (d, $J=8.0 \mathrm{~Hz}, 1 \mathrm{H}$ ), 6.02 (s, 2 H), 3.96 (br s, $4 \mathrm{H}$ ), 3.77 (t, $J=5.0 \mathrm{~Hz}, 4$ H). $\left.{ }^{13} \mathrm{C} \mathrm{NMR} \mathrm{(100} \mathrm{MHz,} \mathrm{CDCl}_{3}\right): \delta=161.2,160.2,155.1,150.1,148.3,147.6,147.4,130.4$, 128.2, 123.5, 113.6, 110.5, 108.2, 101.2, 66.7, 44.4. LRMS: m/z (\%) = $372(33)[\mathrm{M}+2]^{+}, 370$ (100) $[\mathrm{M}]^{+}$. HRMS calcd for $\mathrm{C}_{18} \mathrm{H}_{15} \mathrm{ClN}_{4} \mathrm{O}_{3}$ : 370.0833; found: 370.0851 .

4-(5,8-Di(benzo[d][1,3] dioxol-5-yl)pyrido[4,3-d]pyrimidin-2-yl)morpholine (4g). Yellow solid, mp 300-304 ${ }^{\circ} \mathrm{C}$. IR (KBr): 2893, 1595, 1558, 1518, 1115, 1037, $864 \mathrm{~cm}^{-1} .{ }^{1} \mathrm{H}$ NMR (300 $\mathrm{MHz}_{\mathrm{CDCl}}$ ): $\delta=9.26$ (s, $1 \mathrm{H}$ ), 8.66 (s, $1 \mathrm{H}$ ), 7.36 (s, $\left.1 \mathrm{H},\right), 7.26$ (s, $\left.1 \mathrm{H}\right), 7.23$ (d, $J=8.0 \mathrm{~Hz}, 1$ H), 7.17 (d, $J=8.0 \mathrm{~Hz}, 1 \mathrm{H}), 6.96$ (t, $J=8.0 \mathrm{~Hz}, 2 \mathrm{H}), 6.07$ (s, 2H), 6.04 (s, $2 \mathrm{H}), 3.96$ (br s, 4H), 3.78 (t, $J=4.3 \mathrm{~Hz}, 4 \mathrm{H}) .{ }^{13} \mathrm{C}$ NMR (100 MHz, CF 3 COOD): $\delta=168.5,158.6,156.2,152.5,152.3$, 151.3, 142.1, 129.1, 126.0, 122.3, 118.1, 115.2, 112.4, 112.3, 112.0, 111.3, 105.4, 104.3, 68.1, 48.5. LRMS: $\mathrm{m} / \mathrm{z}(\%)=456(100)[\mathrm{M}]^{+}$.

HRMS calcd for $\mathrm{C}_{25} \mathrm{H}_{20} \mathrm{~N}_{4} \mathrm{O}_{5}$ : 456.1434; found: 456.1441 .

4-(5-Chloro-8-(thiophen-2-yl)pyrido[4,3-d]pyrimidin-2-yl)morpholine (3h). Yellow solid, mp 255-258 ${ }^{\circ} \mathrm{C}$. IR (KBr): 3100, 2864, 1599, 1521, 1117, $717 \mathrm{~cm}^{-1} .{ }^{1} \mathrm{H}$ NMR (300 MHz, $\left.\mathrm{CDCl}_{3}\right)$ : $\delta=9.38$ (s, $1 \mathrm{H}), 8.74$ (s, $1 \mathrm{H}), 7.67$ (d, $J=2.9 \mathrm{~Hz}, 1 \mathrm{H}), 7.50$ (d, $J=4.2 \mathrm{~Hz}, 1 \mathrm{H}), 7.18-7.15$ (m, $1 \mathrm{H}), 4.12$ (br s, $4 \mathrm{H}$ ), 3.85 (br s, $4 \mathrm{H}$ ). ${ }^{13} \mathrm{C}$ NMR (100 MHz, $\mathrm{CDCl}_{3}$ ): $\delta=161.3,160.2$, 153.3, 
149.5, 146.2, 134.8, 128.4, 126.6, 125.5, 124.0, 113.4, 66.8, 44.8. LRMS: m/z (\%) = 334 (33) $[\mathrm{M}+2]^{+}, 332(100)[\mathrm{M}]^{+}$. HRMS calcd for $\mathrm{C}_{15} \mathrm{H}_{13} \mathrm{ClN}_{4} \mathrm{OS}$ : 332.0499; found: 332.0502 .

4-(8-(Benzo $[b]$ thiophen-2-yl)-5-chloropyrido[4,3-d]pyrimidin-2-yl)morpholine (3i). Yellow solid, mp 205-207 ${ }^{\circ} \mathrm{C}$. IR (KBr): 2953, 1600, 1520, 1115, 956, $752 \mathrm{~cm}^{-1} .{ }^{1} \mathrm{H}$ NMR (300 MHz, $\left.\mathrm{CDCl}_{3}\right): \delta=9.37$ (s, $\left.1 \mathrm{H}\right), 8.78$ (s, $\left.1 \mathrm{H}\right), 7.89$ (d, $\left.J=6.5 \mathrm{~Hz}, 1 \mathrm{H}\right), 7.88$ (s, $\left.1 \mathrm{H}\right), 7.82$ (d, $J=8.8$ $\mathrm{Hz}, 1 \mathrm{H}$ ), 7.38-7.35 (m, $2 \mathrm{H}$ ), 4.14 (br s, $4 \mathrm{H}$ ), 3.85 (br s, $4 \mathrm{H}) .{ }^{13} \mathrm{C}$ NMR $\left(100 \mathrm{MHz}, \mathrm{CDCl}_{3}\right.$ ): $\delta=$ 161.3, 160.1, 153.8, 150.5, 147.4, 141.3, 138.9, 135.6, 124.7, 124.4, 124.1, 123.7, 122.6, 121.9, 113.4, 66.8, 44.8. LRMS: $\mathrm{m} / \mathrm{z}(\%)=384(33)[\mathrm{M}+2]^{+}, 382(100)[\mathrm{M}]^{+}$. HRMS calcd for $\mathrm{C}_{19} \mathrm{H}_{15} \mathrm{ClN}_{4} \mathrm{OS}$ : 382.0655; found: 382.0650.

\section{Acknowledgements}

This work was financially supported by the Innovation Program of the Chinese Academy of Sciences (Grant No. KSCX2-YW-R-25), and the National Natural Science Foundation of China (20772138 and 90713034).

\section{References}

1. (a) Rewcastle, G. W.; Murray, D. K.; Elliott, W. L.; Fry, D. W.; Howard, C. T.; Nelson, J. M.; Roberts, B. J.; Vincent, P. W.; Hollis Showalter, H. D.; Winters, R. T.; Denny, W. A. J. Med. Chem. 1998, 41, 742. (b) Anderson, G. L.; Broom, A. D. J. Org. Chem. 1977, 42, 997. (c) Yingling, J. M.; Blanchard, K. L.; Sawyer, J. S. Nat. Rev. Drug Discov. 2004, 3, 1011.

2. (a) Quintela, J. M.; Peinador, C.; Botana, L.; Estévez, M.; Riguera, R. Bioorg. Med. Chem. 1997, 5, 1543. (b) Rosowsky, A.; Chen, H.; Fu, H.; Queener, S. F. Bioorg. Med. Chem. 2003, 11, 59. (c) Duch, D. S.; Edelstein, M. P.; Bowers, S. W.; Nichol, C. A. Cancer Res. 1982, 42, 3987. (d) Lee, C.-H.; Jiang, M.; Cowart, M.; Gfesser, G.; Perner, R.; Kim, K. H.; Gu, Y. G.; Williams, M.; Jarvis, M. F.; Kowaluk, E. A.; Stewart, A. O.; Bhagwat, S. S. J. Med. Chem. 2001, 44, 2133.

3. Hayakawa, M.; Kaizawa, H.; Moritomo, H.; Koizumi, T.; Ohishi, T.; Okada, M.; Ohta, M.; Tsukamoto, S.-i.; Parker, P.; Workman, P.; Waterfield, M. Bioorg. Med. Chem. 2006, 14, 6847.

4. Thompson, A. M.; Murray, D. K.; Elliott, W. L.; Fry, D. W.; Nelson, J. A.; Hollis Showalter, H. D.; Roberts, B. J.; Vincent, P. W.; Denny, W. A. J. Med. Chem. 1997, 40, 3915.

5. Jang, M.-Y.; De Jonghe, S.; Gao, L.-J.; Rozenski, J.; Herdewijn, P. Eur. J. Org. Chem. 2006, 4257.

6. (a) Kotha, S.; Lahiri, K.; Kashinath, D. Tetrahedron 2002, 58, 9633. (b) Littke, A. F.; Dai, C.; Fu, G. C. J. Am. Chem. Soc. 2000, 122, 4020. 
7. (a) Rádl, S.; Hezký, P.; Hafner, W.; Budínský, M.; Hejnová, L. Bioorg. Med. Chem. Lett. 2000, 10, 55. (b) Sahoo, A. K.; Oda, T.; Nakao, Y.; Hiyama, T. Adv. Synth. Catal. 2004, 346, 1715. (c) Dawood, K. M.; Kirschning, A. Tetrahedron 2005, 61, 12121. (d) Desmarets, C.; Omar-Amrani, R.; Walcarius, A.; Lambert, J.; Champagne, B.; Fort, Y.; Schneider, R. Tetrahedron 2008, 64, 372. (e) Ionkin, A. S.; Marshall, W. J.; Fish, B. M. Organometallics 2006, 25, 1461. (f) Na, Y.; Park, S.; Han, S. B.; Han, H.; Ko, S.; Chang, S. J. Am. Chem. Soc. 2004, 126, 250. (g) Lützen , A.; Hapke, M. Eur. J. Org. Chem. 2002, 2292.

8. Fairlamb, I. J. S. Chem. Soc. Rev. 2007, 36, 1036. 